\title{
Increased levels of miR-372 correlate with disease progression in patients with hyperlipidemic acute pancreatitis
}

\author{
YUQIANG SHAN ${ }^{1,2}$, WENCHENG KONG ${ }^{2}$, AKAO ZHU ${ }^{2}$, \\ JIAN ZHANG ${ }^{2}$, RONGCHAO YING ${ }^{2}$ and WEIMING ZHU ${ }^{1}$ \\ ${ }^{1}$ Department of General Surgery, Jinling Clinical Medical College, Nanjing Medical University, Nanjing, \\ Jiangsu 210002; ${ }^{2}$ Department of Gastroenterological Surgery, Affiliated Hangzhou First People's Hospital, \\ Zhejiang University School of Medicine, Hangzhou, Zhejiang 310006, P.R. China
}

Received October 10, 2019; Accepted January 23, 2020

DOI: $10.3892 /$ etm.2020.8609

\begin{abstract}
The aim of the present study was to investigate the expression of microRNA (miRNA)-372 in the serum of patients with hyperlipidemic acute pancreatitis (HTGAP), and its clinical significance. Patients with a serum lipid concentration $\geq 11.3$ or $5.65-11.3 \mathrm{mmol} / 1$ with chylous serum were included in group A $(n=40)$. The remaining patients did not have HTGAP and were included in group B (B). A further 25 patients with hyperlipidemia, but not AP (group C), and 30 healthy volunteers (group D) were recruited as controls. The level of miR-372 in the serum of group A $(4.76 \pm 2.60)$ was significantly increased compared with groups B (0.98 \pm 0.80$), C(0.85 \pm 0.62)$ and D (0.76 \pm 0.44$)$; however, there was no significant difference in the expression of miR-372 between groups B, C and D. The expression level of miR-372 was significantly increased in the severe HTGAP group $(6.45 \pm 2.20)$ compared with the mild HTGAP group $(3.08 \pm 1.74)$. Further experiments suggested that the expression level of miR-372 was positively correlated with the level of triacylglycerol $(\mathrm{r}=0.666 ; \mathrm{P}<0.001)$ but not with the level of amylase $(r=-0.145 ; \mathrm{P}>0.05)$. ROC analysis indicated that the combined use of miR-372 expression levels and Acute Physiology and Chronic Health Evaluation II scoring improved the diagnostic value for HTGAP. In summary, the expression of miR-372 in HTGAP was significantly upregulated and increased with the severity of the disease. The results of the present study may provide a novel strategy for the diagnosis and severity assessment of HTGAP in the clinic.
\end{abstract}

Correspondence to: Dr Weiming Zhu, Department of General Surgery, Jinling Clinical Medical College, Nanjing Medical University, 305 Zhongshan East Road, Nanjing, Jiangsu 210002, P.R. China

E-mail: juwiming@nju.edu.cn

Key words: acute pancreatitis, hyperlipidemia, microRNA-372, diagnosis

\section{Introduction}

Acute pancreatitis (AP) is one of the most common acute abdominal diseases in the clinic worldwide and is typically caused by cholelithiasis, alcoholism and hyperlipidemia $(1,2)$. In recent years, the incidence of hyperlipidemic acute pancreatitis (HTGAP) has been increasing, becoming the secondary cause of AP under biliary AP (3). Patients with HTGAP are prone to recurrence, occasionally with uncontrolled seizures, which seriously endangers the health of the patient $(4,5)$. At present, the diagnosis of HTGAP is primarily based on clinical signs, blood urine amylase levels and serum triacylglycerol levels. However, these diagnostic criteria have a number of limitations, which make the clinical diagnosis and treatment of HTGAP difficult $(6,7)$. Therefore, it is imperative to identify specific biomarkers for the diagnosis of HTGAP.

MicroRNAs (miRNAs/miRs) are a class of endogenous, non-coding small RNAs 19-25 nucleotides in length, which participate in a number of pathophysiological processes, such as cancer and diabetes (8-11). Numerous studies have reported that miRs are closely related to the occurrence and development of AP; therefore, miRs serve as novel markers for the diagnosis and prognosis of the disease (10-12). However, whether miRs can be used as markers for the diagnosis of HTGAP and for predicting the severity of the disease has not been reported.

Abnormal expression of miR-372 has been widely identified in various tumors, including pancreatic adenocarcinoma, as well as ovarian and parathyroid cancer (13-15). It has been reported that miR-372 expression is significantly reduced in human pancreatic adenocarcinoma (13); however, the relationship between miR-372 expression, and the occurrence and development of HTGAP remains unclear. In the present study, the relative expression of miR-372 was detected in 115 patients with AP, and the correlation between miR-372 expression and HTGAP was investigated.

\section{Materials and methods}

Patient samples. Patients with AP were admitted to the Department of General Surgery, Jinling Clinical Medical College between November 2018 and December 2018. 
Clinical data were collected during hospitalization to evaluate the acute physiology and Acute Physiology and Chronic Health Evaluation (APACHE) II score (16) of each patient. Inclusion criteria were as follows: i) Aged between 18 and 65 years; and ii) conformed to the AP diagnostic standard. The AP diagnostic standard consists of i) displaying abdominal pain consistent with AP (acute sudden persistent upper abdominal pain, often radiating to the back); ii) serum amylase and/or lipase levels $\geq 3$ times higher than the upper limit of the normal levels; and iii) imaging results consistent with AP lesions. The following exclusion criteria were used: i) patients with cancer; ii) pregnant patients; iii) patients with psychiatric conditions or patients who refused to cooperate; and iv) patients who give up treatment, transferred out of the department or died.

According to the aforementioned criteria, 115 patients with AP were enrolled (60 male patients and 55 female patients; age, 22-66 years; mean age, $47.3 \pm 11.5$ years). Patients with serum lipid concentrations $\geq 11.3 \mathrm{mmol} / 1$ or serum lipid levels $5.65-11.3 \mathrm{mmol} / \mathrm{l}$ with chylous serum were included in group A $(n=40)$. Subsequently, group A was subdivided into patients with mild (HTG-MAP) or severe (HTG-SAP) HTGAP, according to the AP classification standard $(n=20$ per group). The remainder of the patients with AP did not have HTGAP and were included in group B. A further 25 patients with hyperlipidemia but not AP (group C) admitted to the Department of General Surgery, Jinling Clinical Medical College (China) between November 2018 and December 2018 (12 males and 13 females; age range, 34-68 years; mean age, $48.9 \pm 16.8$ years) and 30 healthy volunteers (group D) from the Physical examination Center, Jinling Clinical Medical College (China) between November 2018 and December 2018 (16 males and 14 females; age range, $42-71$ years; mean age, $46.9 \pm 15.3$ years) were recruited as controls. Computed tomography $(\mathrm{CT})$ images displaying AP status are presented in Fig. 1. The present study was approved by the Ethics Committee of Nanjing Medical University, and written informed consent was obtained from all patients.

Determination of triglyceride and serum amylase levels. $3 \mathrm{ml}$ venous blood was collected from the subjects and placed in heparin anticoagulant tubes. Plasma was detected using an amylase test kit (cat. no. AY7747; Shanghai Zhicheng Biological Technology Co., Ltd.) and triglyceride test kit (cat. no. TR7734; Shanghai Zhicheng Biological Technology Co., Ltd.) using a TBA-120FR automatic biochemical analyzer (Toshiba).

Reverse transcription-quantitative PCR (RT-qPCR). Peripheral blood samples were collected from the patients immediately after admission. Total RNA was extracted from the serum samples using the TRIzol ${ }^{\circledR}$ method (17), and the purity and integrity of the RNA was detected by measuring the optical density (OD) at wavelengths of 260 and $280 \mathrm{~nm}$ using an Orion AquaMate 7000 Vis spectrophotometer (Thermo Fisher Scientific, Inc.). Total RNA was reverse transcribed to cDNA using TaqMan ${ }^{\text {TM }}$ MicroRNA Reverse Transcription kit (Applied Biosystems' Thermo Fisher Scientific, Inc.) according to the manufacturer's instructions. cDNA was stored at $-20^{\circ} \mathrm{C}$ until further analysis. qPCR was performed using the PowerUp SYBR Green master mix (Applied Biosystems; Thermo Fisher Scientific, Inc.) with $1 \mu \mathrm{l}$ cDNA as the template. The following thermocycling conditions were used for the qPCR: Initial denaturation at $95^{\circ} \mathrm{C}$ for $10 \mathrm{sec}$; 39 cycles at $95^{\circ} \mathrm{C}$ for $3 \mathrm{~min}, 95^{\circ} \mathrm{C}$ for $10 \mathrm{sec}$ and $58^{\circ} \mathrm{C}$ for $30 \mathrm{sec}$; and a final extension at $72^{\circ} \mathrm{C}$ for $10 \mathrm{~min}$. miRNA levels were quantified using the $2^{-\Delta \Delta \mathrm{Cq}}$ method (18) and normalized to the internal reference gene U6. qPCR was performed in triplicate wells. The following primer pairs were use for the qPCR: miR-372-RT: 5'-GTCGTATCCAGTGCAGGGTCCGAGGTA TTCGCACTGGATACGACAGAATA-3'; U6-RT: 5'-GTC GTATCCAGTGCAGGGTCCGAGGTATTCGCACTGGAT ACGACAAAATG-3'; miR-372 forward, 5'-GCCCTCAAA TGTGGAGCAC-3'; U6 forward, 5'-GCGCGTCGTGAAGCG TTC-3' and universal reverse primer (Sangon Biotech Co., Ltd.), 5'-GTGCAGGGTCCGAGGT-3'.

Statistical analysis. Data are presented as the mean \pm SD. The unpaired Student's t-test was used to make comparisons between two groups. One-way ANOVA followed by Tukey's post hoc test was used to make comparisons between $>2$ groups. Receiver operating characteristic (ROC) curves were used to assess the value of miR-372 as a biomarker, and the area under the curve (AUC) was reported. Pearson correlation assay demonstrated the level of miR-372 and triglyceride levels, serum amylase levels. Statistical analyses were performed using SPSS software (version 20.0; IBM Corp.). $\mathrm{P}<0.05$ was considered to indicate a statistically significant difference.

\section{Results}

Comparison of general characteristics between the $H T G-M A P$ group and the HTG-SAP group. There was no significant difference in sex or age between the HTG-MAP and HTG-SAP groups. The levels of triacylglycerol in the HTG-SAP group were higher compared with the HTG-MAP group (Table I). Compared with the HTG-MAP group, the levels of CRP were significantly increased in the HTG-SAP group (Table I). The amylase levels in the HTG-SAP group were significantly lower compared with the HTG-MAP group (Table I). Increased levels of serum lipase were also observed in the HTG-SAP group compared with the HTG-MAP group, but the difference was not significant (Table I). Although the APACHE II scores in the HTG-SAP group were higher compared with the HTG-MAP group, the difference was not significant (Table I).

miR-372 expression in the serum of the four different groups. The level of miR-372 expression in the serum of group A (4.76 \pm 2.60$)$ was significantly higher compared with groups B $(0.98 \pm 0.80), C(0.85 \pm 0.62)$ and D (0.76 \pm 0.44$)$; however, there was no significant difference in the expression of miR-372 between groups B, C and D (Fig. 2A). The level of serum miR-372 was significantly increased in the HTG-SAP group $(6.45 \pm 2.20)$ compared with the HTG-MAP group (3.08 \pm 1.74 ; Fig. 2B).

miR-372 expression levels are positively correlated with triglyceride levels in patients with HTGAP. The expression 
Table I. General characteristics of the HTG-MAP group and the HTG-SAP group.

\begin{tabular}{lccr}
\hline Characteristic & HTG-MAP & HTG-SAP & P-value \\
\hline Sex (male/female) & $11 / 9$ & $12 / 8$ & 0.821 \\
Age (years) & $41.3 \pm 3.5$ & $45.6 \pm 3.8$ & 0.436 \\
Triacylglycerol (mmol/l) & $10.2 \pm 2.7$ & $15.9 \pm 4.3$ & 0.075 \\
CRP (mg/l) & $27.4 \pm 21.9$ & $139.8 \pm 101.3$ & 0.015 \\
Amylase (U/l) & $460.5 \pm 132.6$ & $207.9 \pm 45.2$ & 0.021 \\
Lipase (U/l) & $1,177.9 \pm 675.1$ & $1,738.5 \pm 1668.4$ & 0.085 \\
APACHE II score & $4.5 \pm 0.4$ & $12.7 \pm 1.1$ & 0.130 \\
\hline
\end{tabular}

HTG-MAP, mild hyperlipidemic acute pancreatitis; HTG-SAP, severe hyperlipidemic acute pancreatitis; CRP, C-reactive protein; APACHE II, Acute Physiology and Chronic Health Evaluation II.
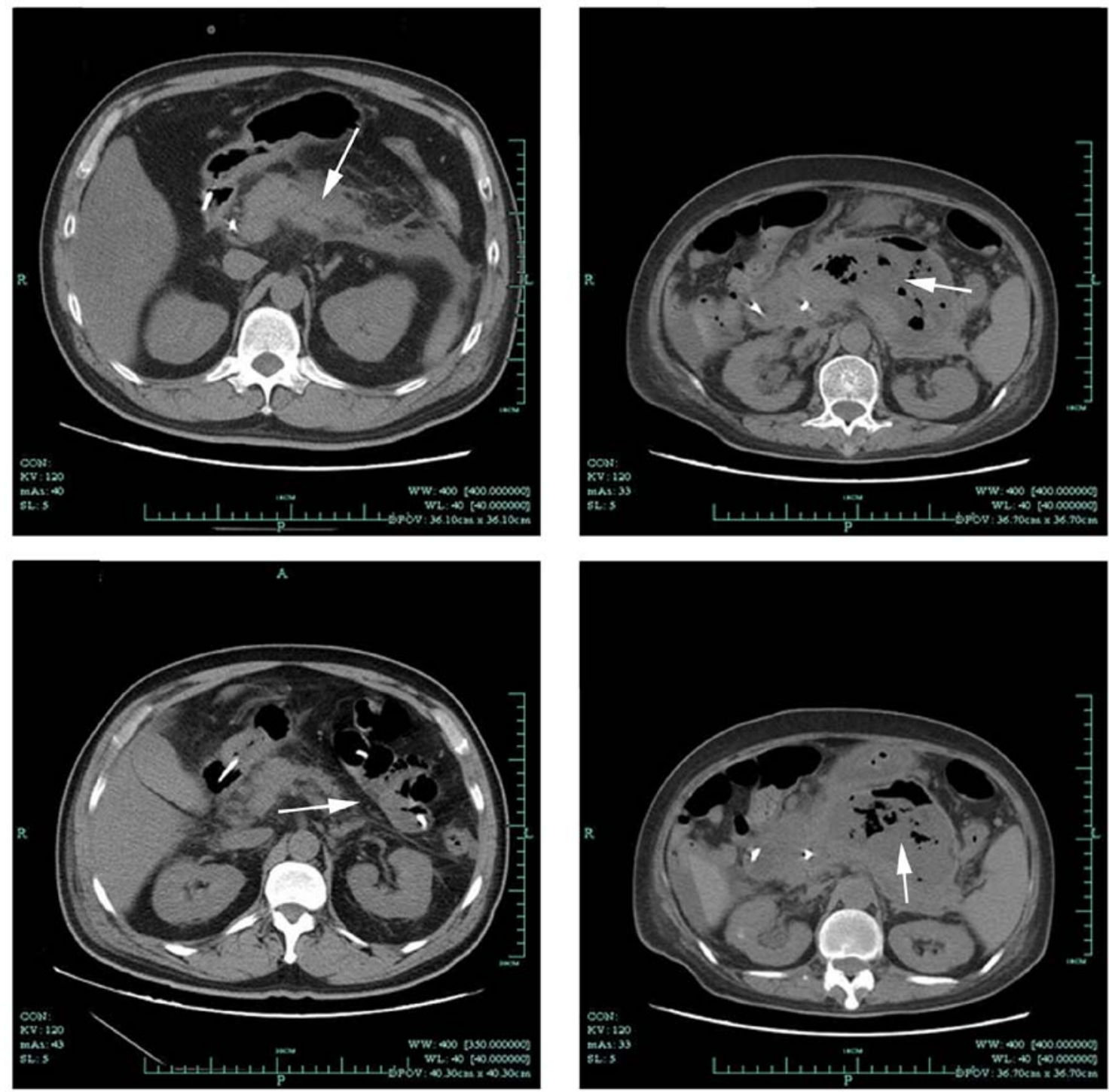

Figure 1. Axial contrast-enhanced computed tomography images of the pancreas of patients with severe AP. The images were obtained from a 42-year-old female patient, a 48 -year-old female, a 47 -year-old male patient and a 52-year-old male patient. White arrows indicate the pancreatic lesions. The patients were discharged without gastrointestinal or intra-abdominal hemorrhage.

of miR-372 was positively correlated with triglyceride levels $(\mathrm{r}=0.666 ; \mathrm{P}<0.001)$, but not with serum amylase levels in patients with HTGAP ( $r=-0.145$; $P>0.05$; Fig. 3 A and $B)$.
Diagnostic value of miR-372 in HTGAP. ROC curve analysis suggested that the AUC for APACHE II scoring, which is used for the diagnosis of HTGAP, was 0.759 with $85.3 \%$ 

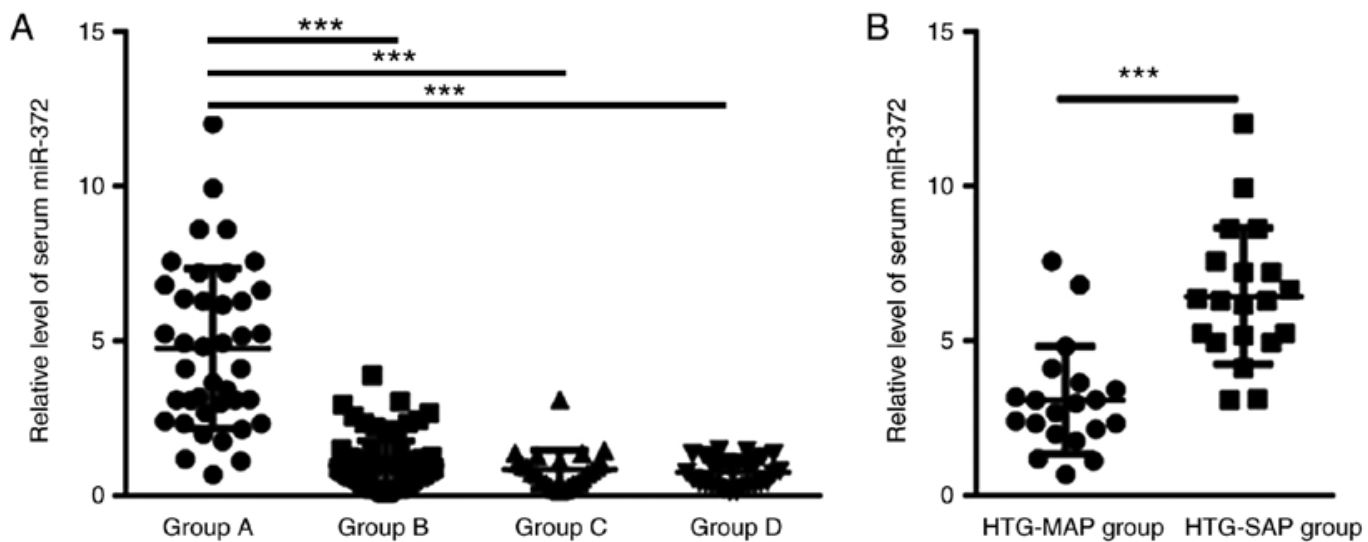

Figure 2. miR-372 expression levels were analyzed in the different groups using RT-qPCR. (A) RT-qPCR analysis suggested that the level of miR-372 expression in the serum of group A was significantly increased compared with groups B, C and D. (B) RT-qPCR analysis suggested that the level of serum miR-372 was significantly increased in the HTG-SAP group compared with the HTG-MAP group. ${ }^{* * *} \mathrm{P}<0.001$ vs. the control. miR, microRNA; RT-qPCR, reverse transcription-quantitative PCR; HTG-SAP, severe hyperlipidemic acute pancreatitis; HTG-MAP, mild hyperlipidemic acute pancreatitis.
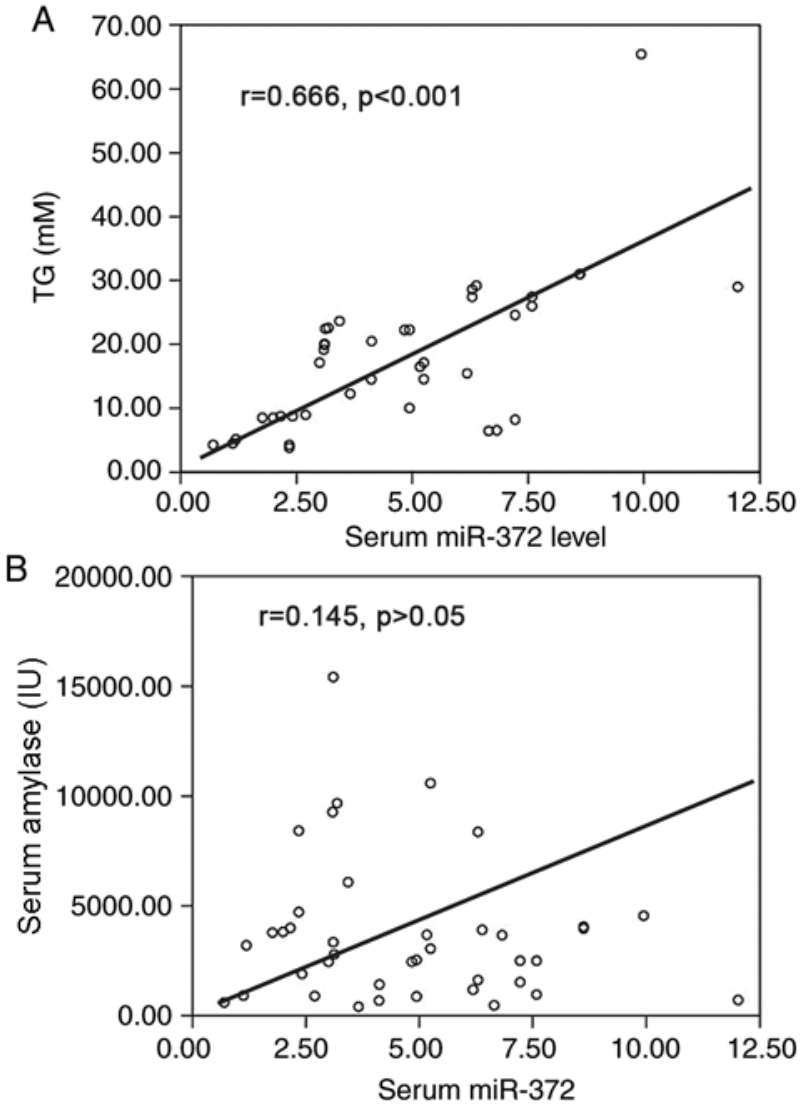

Figure 3. Pearson's correlation coefficient was calculated to analyze the correlation between miR-372 expression levels, and TG and serum amylase levels. miR-371 expression in group A was positively correlated with (A) triglyceride levels, but not with (B) serum amylase levels. miR, microRNA; TG, triglyceride.

sensitivity and $82.1 \%$ specificity (Fig. 4A). Furthermore, the miR-372 expression levels for the diagnosis of HTGAP displayed an AUC of 0.831 with $86.7 \%$ sensitivity and $79.5 \%$ specificity (Fig. 4B). The AUC for the combined use of the APACHE II scoring system and miR-372 expression levels was 0.935 with $89.5 \%$ sensitivity and $87.61 \%$ specificity (Fig. 4C).
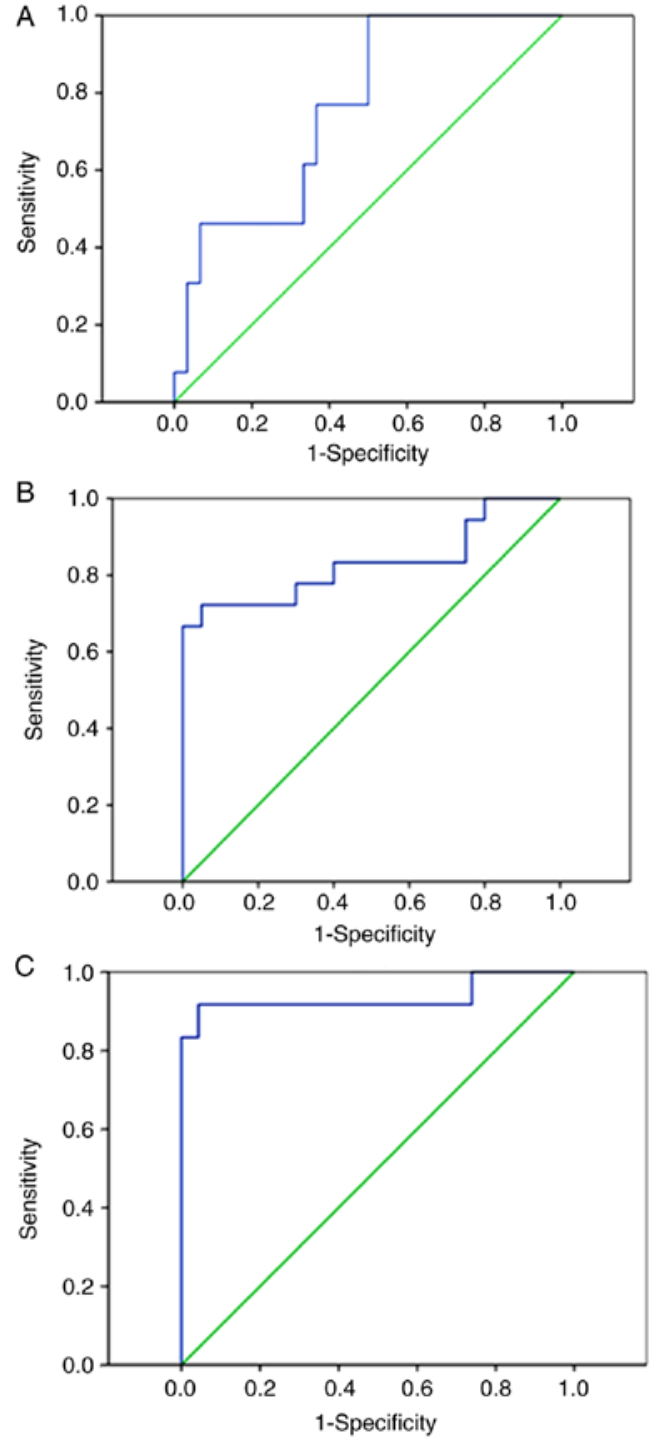

Figure 4. ROC curve analysis suggested that serum miR-372 expression levels could differentiate patients with HTGAP from controls. The AUC of (A) APACHE II score, (B) serum miR-372, and (C) the combined use of APACHE II score and miR-372 were evaluated using ROC curve analysis. ROC, receiver operating characteristic; HTGAP, hyperlipidemic acute pancreatitis; AUC, area under the curve; APACHE II, Acute Physiology and Chronic Health Evaluation II. 


\section{Discussion}

Compared with patients with AP caused by other factors, including, gallstones and alcohol consumption, patients with HTGAP are more prone to recurrence, systemic inflammatory response syndrome (SIRS) and multiple organ failure (MOF), and display high mortality and poor prognosis (19-21). At present, the clinical diagnosis of HTGAP primarily relies on serological and imaging examinations $(22,23)$. Serological examination is typically based on triacylglycerol and amylase levels (24); however, since triacylglycerol often interferes with amylase measurements, increases in amylase in patients with HTGAP are not obvious, which makes it easy for the diagnosis of HTGAP to be missed in the clinic $(25,26)$.

In recent years, a number of studies have reported that miRs are closely related to the occurrence and development of AP $(11,27)$; however, the role of miRs in HTGAP has not been extensively reported. In the present study, RT-qPCR results suggested that the level of miR-372 in the serum of patients with HTGAP was significantly increased compared with patients who did not have HTGAP, indicating that the expression of miR-372 was abnormally high in patients with HTGAP. Therefore, abnormally high miR-372 expression may serve as a reference index for the diagnosis of HTGAP. ROC curve analysis indicated that miR-372 had diagnostic value for HTG-SAP, with an AUC of 0.831. The results suggested that miR-372 may serve as a specific marker for HTGAP, which may provide a novel strategy for the clinical diagnosis of HTGAP. However, the sample size of the present study was limited; therefore, further investigation using larger sample sizes is required to support the use of miR-371 for the clinical diagnosis of HTGAP.

Early prediction of disease severity is also important for the treatment and prognostic evaluation of HTGAP (28), as the progression of HTGAP is often rapid. Distinguishing HTG-MAP and HTG-SAP is important for the treatment and control of HTGAP (29). At present, the most common methods used to predict AP are the APACHE II and Ranson scoring systems (30). These scores often need to be evaluated within 24 or $48 \mathrm{~h}$ of admission, and involve numerous steps and a complicated process (30). In the present study, ROC curve analysis of the APACHE II score and miR-372 expression was performed, and the AUC was 0.759 and 0.831 , respectively. Both methods displayed good sensitivity and specificity, suggesting that miR-372 could be used as a predictor of AP severity.

In addition, the present study examined the correlation between miR-372 expression, and triacylglycerol and amylase levels. The results suggested that the expression of serum miR-372 in HTGAP was positively correlated with triacylglycerol levels, but not with amylase levels. A possible explanation for the result is that in the hypertriglyceride environment, amylase, which is the most commonly used indicator of AP, inaccurately appears to be at normal levels (31).

The present study had a number of limitations. Firstly, the sample size was relatively small. Secondly, it is important to include other types of patients to validate the specificity of abnormal miR-372 expression, since abnormal expression of serum miR-372 is also present in other diseases. Thirdly, as a potential biomarker, it is important to evaluate the combined use of serum miR-372 and existing indicators, which may improve the specificity of the diagnosis in patients with HTGAP.

In conclusion, the expression of miR-372 in HTGAP was significantly upregulated and increased with the severity of the disease. The results of the present study suggested a novel strategy for the diagnosis and severity assessment of HTGAP in the clinic.

\section{Acknowledgements}

Not applicable.

\section{Funding}

The present study was supported by the Zhejiang Public Welfare Technology Application Research Project (grant no. 2016C33SA100062).

\section{Availability of data and materials}

The datasets used and/or analyzed in the current study are available from the corresponding author on reasonable request.

\section{Authors' contributions}

YS performed the experiments, analyzed the data and wrote the manuscript. WK, AZ, JZ and RY performed the RT-qPCR experiments. WZ designed the study, analyzed the data and gave final approval of the manuscript to be published. All authors read and approved the final manuscript.

\section{Ethics approval and consent to participate}

The present study was approved by the Ethics Committee of Nanjing Medical University. All patients provided written informed consent.

\section{Patient consent for publication}

All patients provided written informed consent for the publication of their data in the present study.

\section{Competing interests}

The authors declare that they have no competing interests.

\section{References}

1. Brown TT and Prahlow JA: Postmortem serum amylase and lipase analysis in the diagnosis of acute pancreatitis. Acad Forensic Pathol 8: 311-323, 2018.

2. Cao X, Wang HM, Du H, Chen EX, Yang XF, Wang SL, Ding Y and She ZF: Early predictors of hyperlipidemic acute pancreatitis. Exp Ther Med 16: 4232-4238, 2018.

3. Dolay K, Hasbahceci M, Hatipoglu E, Ümit Malya F and Akçakaya A: Endoscopic diagnosis and treatment of biliary obstruction due to acute cholangitis and acute pancreatitis secondary to Fasciola hepatica infection. Ulus Travma Acil Cerrahi Derg 24: 71-73, 2018.

4. Durmaz MS, Arslan S, Özbakır B, Güngör G, Tolu İ, Arslan FZ, Sivri M and Koplay M: Effectiveness of Shear Wave Elastography in the diagnosis of acute pancreatitis on admission. Med Ultrason 20: 278-284, 2018. 
5. Charlesworth A, Steger A and Crook MA: Hyperlipidemic acute pancreatitis and the Apolipoprotein E4 allele. Pancreas 46: e3-e4, 2017.

6. French JM, Twedt DC, Rao S and Marolf AJ: Computed tomographic angiography and ultrasonography in the diagnosis and evaluation of acute pancreatitis in dogs. J Vet Intern Med 33: 79-88, 2019.

7. Durval A, Zamidei L, Bettocchi D, Luzzio MG and Consales G: Hyperlipidemic acute pancreatitis: A possible role of antiretroviral therapy with entecavir. Minerva Anestesiol 77: 1018-1021, 2011.

8. Noorolyai S, Baghbani E, Aghebati Maleki L, Baghbanzadeh Kojabad A, Shanehbansdi D, Khaze Shahgoli V, Mokhtarzadeh A and Baradaran B: Restoration of miR-193a-5p and miR-146 a-5p expression induces G1 arrest in colorectal cancer through targeting of MDM2/p53. Adv Pharm Bull 10: $130-134,2020$

9. Zhao C, Fei X, Xu B, Lu Y and Zhang Q: Long non-coding RNA HEIH contributes to diabetic retinopathy by regulating miR-939/ VEGF axis. Int J Clin Exp Pathol 12: 2022-2033, 2019.

10. Lu P, Wang F, Wu J, Wang C, Yan J, Li ZL, Song JX and Wang JJ: Elevated Serum miR-7, miR-9, miR-122, and miR-141 are noninvasive biomarkers of acute pancreatitis. Dis Markers 2017: 7293459, 2017.

11. Miao B, Qi WJ, Zhang SW, Wang H, Wang C, Hu L, Huang GW, Li SR and Wang H: miR-148a suppresses autophagy by down-regulation of IL-6/STAT3 signaling in cerulein-induced acute pancreatitis. Pancreatology 19: 557-565, 2019.

12. Dixit AK, Sarver AE, Yuan Z, George J, Barlass U, Cheema H, Sareen A, Banerjee S, Dudeja V, Dawra R, et al: Comprehensive analysis of microRNA signature of mouse pancreatic acini: Overexpression of miR-21-3p in acute pancreatitis. Am J Physiol Gastrointest Liver Physiol 311: G974-G980, 2016.

13. Chen H, Zhang Z, Lu Y, Song K, Liu X, Xia F and Sun W: Downregulation of ULK1 by microRNA-372 inhibits the survival of human pancreatic adenocarcinoma cells. Cance Sci 108: 1811-1819, 2017

14. Guan X, Zong ZH, Chen S, Sang XB, Wu DD, Wang LL, Liu Y and Zhao Y: The role of miR-372 in ovarian carcinoma cell proliferation. Gene 624: 14-20, 2017.

15. Verdelli C, Forno I, Morotti A, Creo P, Guarnieri V, Scillitani A, Cetani F, Vicentini L, Balza G, Beretta E, et al: The aberrantly expressed miR-372 partly impairs sensitivity to apoptosis in parathyroid tumor cells. Endocr Relat Cancer 25: 761-771, 2018.

16. Yuan S, Gao Y, Ji W, Song J and Mei X: The evaluation of acute physiology and chronic health evaluation II score, poisoning severity score, sequential organ failure assessment score combine with lactate to assess the prognosis of the patients with acute organophosphate pesticide poisoning. Medicine (Baltimore) 97 e10862, 2018

17. Wang D, Guo C, Kong T, Mi G, Li J and Sun Y: Serum miR-22 may be a biomarker for papillary thyroid cancer. Oncol Lett 17: $3355-3361,2019$.
18. Livak KJ and Schmittgen TD: Analysis of relative gene expression data using real-time quantitative PCR and the 2(-Delta Delta C(T)) method. Methods 25: 402-408, 2001.

19. Jagannath S and Garg PK: Recurrent acute pancreatitis: Current concepts in the diagnosis and management. Curr Treat Options Gastroenterol 16: 449-465, 2018.

20. Jin DX, Lacson R, Cochon LR, Alper EC, McNabb-Baltar J, Banks PA and Khorasani R: A clinical model for the early diagnosis of acute pancreatitis in the emergency department. Pancreas 47: 871-879, 2018.

21. Hang Y, Chen Y, Lu LX and Zhu CQ: Acute hyperlipidemic pancreatitis in a pregnant woman. World J Emerg Med 4: 311-313, 2013.

22. Kaya M, Değirmenci S, Göya C, Tuncel ET, Uçmak F and Kaplan MA: The importance of acoustic radiation force impulse (ARFI) elastography in the diagnosis and clinical course of acute pancreatitis. Turk J Gastroenterol 29: 342-347, 2018.

23. Huang YX, Jia L, Jiang SM, Wang SB, Li MX and Yang BH: Incidence and clinical features of hyperlipidemic acute pancreatitis from Guangdong, China: A retrospective multicenter study. Pancreas 43: 548-552, 2014.

24. Lopes CV, Pereira-Lima J and Hartmann AA: The role of linear endosonography for the diagnosis of acute pancreatitis when other methods failed. Clin Res Hepatol Gastroenterol 43: 98-103, 2019.

25. Senates E: Toward a new ultrasound-based imaging method for the diagnosis of acute pancreatitis: A preliminary study suggesting that it may be feasible. Turk J Gastroenterol 29: $256-258,2018$

26. Li MQ, Shi ZX, Xu JY, Lu B, Li JQ, Xu YJ, Wang XM, Li SM and Mo X: Hemodiafiltration combined with resin-mediated absorption as a therapy for hyperlipidemic acute pancreatitis. Cell Biochem Biophys 69: 699-702, 2014.

27. Li X, Lin Z, Wang L, Liu Q, Cao Z, Huang Z, Zhong M, Peng S, Zhang Y, Li Y and Ma X: RNA-Seq analyses of the role of miR-21 in acute pancreatitis. Cell Physiol Biochem 51: 2198-2211, 2018.

28. Zhang Y, Yan L and Han W: Elevated level of miR-551b-5p is associated with inflammation and disease progression in patients with severe acute pancreatitis. Ther Apher Dial 22: 649-655, 2018.

29. Lu J, Xie Y, Du J, Kang M, Jin W, Li Y, Xie H, Cheng R, Tian R and Wang R: Penta-therapy for severe acute hyperlipidemic pancreatitis. Am J Emerg Med 36: 1789-1795, 2018.

30. Markota A, Knehtl M, Sinkovic A, Ekart R, Hojs R and Bevc S Plasma exchange treatment for acute hyperlipidemic pancreatitis with falsely low levels of serum triglycerides-a case report. Transfus Apher Sci 51: 178-180, 2014

31. Yang L, Zhao Z, Zhou K and Zhang Y: Acute hyperlipidemic pancreatitis accompanied by chylous ascites with normal amylase and lipase in pregnancy. J Clin Lipidol 11: 1091-1094, 2017. 